\title{
Criteria for H-Matrices Based on $\gamma$-Diagonally Dominant Matrix
}

\author{
Xin $\operatorname{Li}^{1} \&$ Mei Qin ${ }^{1}$ \\ ${ }^{1}$ College of Science, University of Shanghai for Science and Technology, Shanghai, China \\ Correspondence: Mei Qin, College of Science, University of Shanghai for Science and Technology, Shanghai, 200093, \\ China. E-mail: 1xmath@163.com
}

Received: September 16, 2019 Accepted: October 11, 2019 Online Published: October 28, 2019

doi:10.5539/jmr.v11n6p1

URL: https://doi.org/10.5539/jmr.v11n6p1

\begin{abstract}
In this paper, we present a new practical criteria for H-matrix based on $\gamma$-diagonally dominant matrix. In order to make the judgment conditions convenient and effective, we give two new definitions, one is called strong and weak diagonally dominant degree, the other is called the sum of non-principal diagonal element for the matrix. Further, we obtain a new practical method for the determination of the H-matrix by combining the properties of $\gamma$-diagonally dominant matrix, constructing positive diagonal matrix, and adding the appropriate parameters. Finally, we offer numerical examples to verify the validity of the judgment conditions, corresponding numerical examples compared the new criteria and the existing results are presented to verify the advantages of the new determination method.
\end{abstract}

Keywords: H-matrix, $\gamma$-diagonally dominant matrix, irreducible matrix, strong and weak diagonally dominant degree

\section{Introduction}

H-matrix is an important concept in computational mathematics. The definition of H-matrix was first given by A.M. Ostrowski in 1937 (Ostrowski,1937). As a typical special matrix, H-matrix not only has important theoretical research value, but also has wide application in many fields such as numerical algebra, statistics and economic mathematics, etc (Gan, \& Huang, 2004). For a long time, researchers' enthusiasm for the study of the properties of H-matrix, especially the criterion of H-matrix, has not diminished. For instance, Varga R.S. (Varga, 1976), Gao Y.M. (Gao, \& Wang, 1996), Cvetkovic L. (Cvetkovic, \& Kostic, 2005) gave some direct judgement methods of H-matrix by dividing matrix index sets and scaling inequalities. Huang T. Z. (Huang, Leng, Wachspress, \& Tang, 2004), Liu J.Z. (Liu, \& Huang, 2004) and other scholars have presented the relationship between strictly diagonally dominant matrices and H-matrices. In recent years, scholars such as Xie Q.M., He A.Q. (Xie, He, \& Liu, 2007), Gao H.S. (Gao, \& Han, 2017), Jeribi A. (Jeribi, Krichen, \& Zitouni, 2019)have given some iterative discriminant algorithms for H-matrices on the basis of the existing theory. These algorithms speed up the determination and expand the scope of discrimination. However, until now, it has been still not convenient to check if a given matrix is an H-matrix.

Consider the structure of stationary linear large-scale systems

$$
\frac{d X}{d t}=A X
$$

where $A=\left(a_{i j}\right) \in \mathbb{C}^{n \times n}, X=\left(x_{1}, x_{2}, \cdots, x_{n}\right)^{T} \in \mathbb{C}^{n}$.

For one thing, it is well known that $\mathrm{H}$-matrices play an important role in matrix theory, numerical analysis, economic mathematics, etc ( $\mathrm{Li}, \&$ Sun, 2009). For example, solving the linear equation $A x=b$, when the coefficient matrix A is H-matrix, then many classical iterative methods such as Jacobi, Gaussłseidel, SOR and their improved algorithms are convergent. Hence, how to judge H-matrices is important and necessary.

For another, in the 1960s, people defined a kind of matrix class - generalized strictly diagonally dominant matrix class (Zhu, 1995), which is completely equivalent to H-matrix class in essence mathematics from different angles and different problem backgrounds. With the later $\gamma$-diagonally dominant matrix, $\gamma$-chain diagonally dominant matrix and other concepts were proposed (Liu, \& Huang, 2010), we find that the properties of $\gamma$-diagonally dominant matrix is very ingenious for judging $\mathrm{H}$-matrix conditions. Hence, how to determine $\mathrm{H}$-matrix by using the properties of $\gamma$-diagonally dominant matrix is very useful.

Based on the above, studying the properties of $\gamma$-diagonally dominant matrix and seeking for the practical criterion of H-matrix may have important theoretical and practical significance. In this paper, we first present two new definitions, one is called strong and weak diagonally dominant degree, the other is called the sum of non-principal diagonal element 
for the matrix. Compared with the pervious definitions such as diagonally dominant degree, they are not only more helpful, but also more general. Then, using two new definitions, combining the properties of $\gamma$-diagonally dominant matrix, constructing positive diagonal matrices and adding appropriate parameters, we obtain the criterion of H-matrix. It may be used to judge H-matrix more faster than several known methods in pervious article. Finally, we give numerical examples to verify the validity and illustrate the advantages of the criteria of nonsingular H-matrices.

In order to state the main results, we need the following symbols. Let $\mathbb{C}^{n \times n}\left(\mathbb{R}^{n \times n}\right)$ denote the set of all $n \times n$ complex(real) matrices, $N=\{1,2, \cdots, n\}$ and $A=\left(a_{i j}\right) \in \mathbb{C}^{n \times n}$. Denote $|A|=\left(\left|a_{i j}\right|\right)$ and $\forall i, j \in N$,

$$
R_{i}=\sum_{j \in N, j \neq i}\left|a_{i j}\right|, Q_{i}=\sum_{j \in N, j \neq i}\left|a_{j i}\right| .
$$

Now, we introduce some notations

$$
\begin{aligned}
& N_{1}=\left\{i|0<| a_{i i} \mid=\gamma R_{i}+(1-\gamma) Q_{i}\right\}, \\
& N_{2}=\left\{i|0<| a_{i i} \mid<\gamma R_{i}+(1-\gamma) Q_{i}\right\}, \\
& N_{3}=\left\{i \| a_{i i} \mid>\gamma R_{i}+(1-\gamma) Q_{i}>0\right\} .
\end{aligned}
$$

Obviously, $N=N_{1} \cup N_{2} \cup N_{3}$.

\section{Definitions and Lemmas}

Definition 2.1 (Liu, Zhang, Zhou, \& Tu, 2018) Let $A=\left(a_{i j}\right) \in \mathbb{R}^{n \times n}$, if $a_{i j} \leq 0$ for all $i \neq j$, then A is said to be a Z-matrix. If $A$ can be expressed in the form

$$
A=s I_{n}-B, s \geq 0, B \geq 0,
$$

where $B \geq 0$, and $s \geq \rho(B), I_{n}$ denotes a unit matrix of order $\mathrm{n}, \rho(B)$ stands for the spectral radius of $\mathrm{B}$, then A is said to be an M-matrix.

Definition 2.2 ( $\mathrm{Li}$, Liu, \& Zhang, 2017) Let $A=\left(a_{i j}\right) \in \mathbb{C}^{n \times n}$, denoted by $\mu(A)=\left(t_{i j}\right) \in \mathbb{R}^{n \times n}$, is defined by

$$
t_{i j}=\left\{\begin{array}{r}
\left|a_{i j}\right|, i=j, \\
-\left|a_{i j}\right|, i \neq j,
\end{array}\right.
$$

then $\mu(A)$ is said to be a comparison matrix of matrix A. If $\mu(A) \in M_{n}$, then A is called a general H-matrix.

Definition 2.3 Let $A=\left(a_{i j}\right) \in \mathbb{C}^{n \times n}$. If for $i \in N$, such that

$$
\left|a_{i i}\right| \geq R_{i}(i \in N),
$$

then $\mathrm{A}$ is said to be a diagonally dominant matrix and is denoted by $A \in D_{0}$.

Definition 2.4 Let $A=\left(a_{i j}\right) \in \mathbb{C}^{n \times n}$. If $\exists \gamma \in[0,1]$, for $\forall i \in N$, such that

$$
\left|a_{i i}\right| \geq \gamma R_{i}+(1-\gamma) Q_{i},
$$

then $\mathrm{A}$ is said to be a $\gamma$-diagonally dominant matrix and is denoted by $A \in D_{\gamma_{0}}$. If for $i \in N$, such that

$$
\left|a_{i i}\right|>\gamma R_{i}+(1-\gamma) Q_{i},
$$

then A is said to be a strictly $\gamma$-diagonally dominant matrix and is denoted by $A \in D_{\gamma}$.

Lemma 2.1 (Gao, \& Wang, 1996) Let $A=\left(a_{i j}\right) \in \mathbb{C}^{n \times n}$. If there exists positive diagonally matrix $X=\operatorname{diag}\left(d_{1}, d_{2}, \cdots, d_{n}\right)$, where $d_{i}>0, i \in N$, such that

$$
A X \in S D,
$$

then $\mathrm{A}$ is said to be a generalized strictly diagonally dominant matrix and is denoted by $A \in G S D$.

Lemma 2.2 ( $\mathrm{Liu}, \& \mathrm{He}, 2007) \mathrm{A}$ is an H-matrix if and only if A is a generalized strictly diagonally dominant matrix.

Lemma 2.3 (Gan, \& Huang, 2004) Let $A=\left(a_{i j}\right) \in \mathbb{C}^{n \times n}$ be $\gamma$-diagonally dominant matrix. If A satisfies either

(i) A is an irreducible matrix, $N_{\gamma}(A)=\left\{i \in N \| a_{i i} \mid>\gamma R_{i}+(1-\gamma) Q_{i}\right\} \neq \varnothing$;

(ii) $N_{\gamma}(A)=\left\{i \in N|| a_{i i} \mid>\gamma R_{i}+(1-\gamma) Q_{i}\right\} \neq \varnothing$ and $\forall i \in N-N_{\gamma}(A)$; 
there must exists a nonzero elements chain $a_{i i_{1}}, a_{i_{1} i_{2}}, \cdots, a_{i_{s} i_{m}}$, and $i \neq i_{1}, i_{1} \neq i_{2}, \cdots, i_{s} \neq i_{m}, i_{m} \in N_{\gamma}(A)$, then $\mathrm{A}$ is a generalized strictly $\gamma$-diagonally dominant matrix.

If $N_{2}=\varnothing$, then A is a strictly $\gamma$-diagonally dominant matrix; If $N_{3}=\varnothing$, then A is not a strictly $\gamma$-diagonally dominant matrix.

So, we always assume that $N_{2}, N_{3} \neq \varnothing$ and all diagonal entries of A are nonzero.

\section{Main Results}

In this section, we propose two new definitions and present new judgment conditions for H-matrices. In order to verify the feasibility of judging conditions, we give a numerical example.

Definition 3.1 Let $A=\left(a_{i j}\right) \in \mathbb{C}^{n \times n}$. Then A is a $\gamma$-diagonally dominant matrix, if there exists $\gamma \in[0,1]$, such that

$$
\left|a_{i i}\right| \geq \gamma R_{i}+(1-\gamma) Q_{i}, \forall i \in N,
$$

we denote $S_{i}=\gamma R_{i}+(1-\gamma) Q_{i}$ as the sum of non-principal diagonal element for the matrix A. If $\gamma=0$, such that $S_{i}=Q_{i}$,

$$
\left|a_{i i}\right|>Q_{i},
$$

then $S_{i}$ is said to be column sum of matrix A.

If $\gamma=1$, such that $S_{i}=R_{i}$,

$$
\left|a_{i i}\right|>R_{i},
$$

then $S_{i}$ is said to be row sum of matrix A.

Definition 3.2 Let $A=\left(a_{i j}\right) \in \mathbb{C}^{n \times n}$. If exists $\omega_{i}$, for $\forall i \in N_{2}$, such that

$$
\omega_{i}=\frac{S_{i}-\left|a_{i i}\right|}{S_{i}},
$$

we called $\omega_{i}$ the weak diagonally dominant degree of matrix A.

If exists $\mu_{j}$, for $\forall j \in N_{3}$, such that

$$
\mu_{j}=\frac{S_{j}}{\left|a_{j j}\right|},
$$

we called $\mu_{j}$ the strong diagonally dominant degree of matrix A.

Theorem 3.1 Let $A=\left(a_{i j}\right) \in \mathbb{C}^{n \times n}$, if $N_{1}, N_{2}, N_{3}$ satisfy $N=N_{1} \cup N_{2} \cup N_{3}$, then for $i \in N_{2}, j \in N_{3}$,

$$
\begin{gathered}
\left(\omega_{i}\left|a_{i i}\right|-\left[\gamma \sum_{t \in N_{1}, t \neq i}\left|a_{i t}\right|+\gamma \omega_{t} \sum_{t \in N_{2}, t \neq i}\left|a_{i t}\right|+\omega_{i}(1-\gamma) Q_{i}\right]\right)\left(S_{j}-\left[\mu_{t} \gamma \sum_{t \in N_{3}, t \neq j}\left|a_{j t}\right|+\mu_{j}(1-\gamma) Q_{j}\right]\right) \\
>\left(\mu_{t} \gamma \sum_{t \in N_{3}, t \neq i}\left|a_{i t}\right|\right)\left(\gamma \sum_{t \in N_{1}, t \neq j}\left|a_{j t}\right|+\gamma \omega_{t} \sum_{t \in N_{2}, t \neq j}\left|a_{j t}\right|\right),
\end{gathered}
$$

then $\mathrm{A}$ is called an H-matrix.

Proof. Note that

$$
\begin{gathered}
d^{(1)}=\min \frac{\omega_{i}\left|a_{i i}\right|-\gamma \sum_{t \in N_{1}, t \neq i}\left|a_{i t}\right|-\gamma \omega_{t} \sum_{t \in N_{2}, t \neq i}\left|a_{i t}\right|-\omega_{i}(1-\gamma) Q_{i}}{\mu_{t} \gamma \sum_{t \in N_{3}, t \neq i}\left|a_{i t}\right|}, \\
d^{(2)}=\max \frac{\gamma\left(\sum_{t \in N_{1}, t \neq j}\left|a_{j t}\right|+\omega_{t} \sum_{t \in N_{2}, t \neq j}\left|a_{j t}\right|\right)}{S_{j}-\left[\mu_{t} \gamma \sum_{t \in N_{3}, t \neq j}\left|a_{j t}\right|+\mu_{j}(1-\gamma) Q_{j}\right]} .
\end{gathered}
$$

We can get $d^{(1)}>d^{(2)}$, then we take any $\mathrm{k}$ and assume that $\min d^{(1)} \geq k \geq \max d^{(2)}$, we suppose a positive diagonally matrix

$$
X=\operatorname{diag}\left\{d_{1}, d_{2}, \cdots, d_{n}\right\},
$$

where

$$
d_{i}=\left\{\begin{array}{r}
1, i \in N_{1} \\
\omega_{i}, i \in N_{2} \\
k \mu_{i}, i \in N_{3}
\end{array}\right.
$$

Let $B=\left(b_{i j}\right)=A X$, then we prove that $\mathrm{B}$ is a strictly $\gamma$-diagonally dominant matrix. 
(i) For $\forall i \in N_{1}$,

it is clear that $\left|a_{i i}\right|=\left|b_{i i}\right|$, we infer from the irreducibility of matrix A that

$$
\sum_{t \in N_{2}, t \neq i}\left|a_{i t}\right| \neq 0
$$

or

$$
\sum_{t \in N_{3}, t \neq i}\left|a_{i t}\right| \neq 0
$$

thus, we know that

$$
\gamma R_{i}(B)+(1-\gamma) Q_{i}(B)=\gamma\left(1 \cdot \sum_{t \in N_{1}, t \neq i}\left|a_{i t}\right|+\omega_{t} \cdot \sum_{t \in N_{2}, t \neq i}\left|a_{i t}\right|+k \mu_{t} \cdot \sum_{t \in N_{3}, t \neq i}\left|a_{i t}\right|\right)+(1-\gamma) Q_{i},
$$

then, we have

$$
\left|a_{i i}\right|=\gamma\left(\sum_{t \in N_{1}, t \neq i}\left|a_{i t}\right|+\sum_{t \in N_{2}, t \neq i}\left|a_{i t}\right|+\sum_{t \in N_{3}, t \neq i}\left|a_{i t}\right|\right)+(1-\gamma) Q_{i},
$$

thus, we arrive that

$$
\left|b_{i i}\right|=\left|a_{i i}\right| \geq \gamma R_{i}(B)+(1-\gamma) Q_{i}(B)
$$

(ii) For $\forall i \in N_{2}, k<d^{(1)}$,

we have

$$
\gamma k \mu_{t} \sum_{t \in N_{3}, t \neq i}\left|a_{i t}\right| \leq \omega_{i}\left|a_{i i}\right|-\gamma \sum_{t \in N_{1}, t \neq i}\left|a_{i t}\right|-\gamma \omega_{t} \sum_{t \in N_{2}, t \neq i}\left|a_{i t}\right|-\omega_{i}(1-\gamma) Q_{i},
$$

then, we know that

$$
\gamma R_{i}(B)+(1-\gamma) Q_{i}(B)=\gamma\left(1 \cdot \sum_{t \in N_{1}, t \neq i}\left|a_{i t}\right|+\omega_{t} \cdot \sum_{t \in N_{2}, t \neq i}\left|a_{i t}\right|+k \mu_{t} \cdot \sum_{t \in N_{3}, t \neq i}\left|a_{i t}\right|\right)+\omega_{i}(1-\gamma) Q_{i} .
$$

Thus, we have

$$
\begin{aligned}
\left|b_{i i}\right|=\omega_{i}\left|a_{i i}\right| & =\gamma \sum_{t \in N_{1}, t \neq i}\left|a_{i t}\right|+\gamma \omega_{t} \sum_{t \in N_{2}, t \neq i}\left|a_{i t}\right|+\omega_{i}(1-\gamma) Q_{i} \\
& +\omega_{i}\left|a_{i i}\right|-\left[\gamma\left(\sum_{t \in N_{1}, t \neq i}\left|a_{i t}\right|+\omega_{t} \sum_{t \in N_{2}, t \neq i}\left|a_{i t}\right|\right)+\omega_{i}(1-\gamma) Q_{i}\right],
\end{aligned}
$$

we infer from (3.4) that

$$
\left|b_{i i}\right|=\omega_{i}\left|a_{i i}\right| \geq \gamma R_{i}(B)+(1-\gamma) Q_{i}(B) .
$$

(iii) For $\forall i \in N_{3}$, since $k>\operatorname{maxd}(2)$,

we get

$$
k\left(S_{i}-\left[\mu_{t} \gamma \sum_{t \in N_{3}, t \neq i}\left|a_{i t}\right|+\mu_{j}(1-\gamma) Q_{i}\right]\right) \geq \gamma\left(\sum_{t \in N_{1}, t \neq i}\left|a_{i t}\right|+\omega_{t} \sum_{t \in N_{2}, t \neq i}\left|a_{i t}\right|\right),
$$

then

$$
k\left(S_{i}-\mu_{t} \gamma \sum_{t \in N_{3}, t \neq i}\left|a_{i t}\right|\right)-k \mu_{j}(1-\gamma) Q_{i} \geq \gamma\left(\sum_{t \in N_{1}, t \neq i}\left|a_{i t}\right|+\omega_{t} \sum_{t \in N_{2}, t \neq i}\left|a_{i t}\right|\right),
$$

it is clear that

$$
k\left(S_{i}-\mu_{t} \gamma \sum_{t \in N_{3}, t \neq i}\left|a_{i t}\right|\right)-\gamma\left(\sum_{t \in N_{1}, t \neq i}\left|a_{i t}\right|+\omega_{t} \sum_{t \in N_{2}, t \neq i}\left|a_{i t}\right|\right) \geq k \mu_{j}(1-\gamma) Q_{i},
$$


we know that

$$
\gamma R_{i}(B)+(1-\gamma) Q_{i}(B)=\gamma\left(1 \cdot \sum_{t \in N_{1}, t \neq i}\left|a_{i t}\right|+\omega_{t} \cdot \sum_{t \in N_{2}, t \neq i}\left|a_{i t}\right|+k \mu_{t} \cdot \sum_{t \in N_{3}, t \neq i}\left|a_{i t}\right|\right)+\mu_{j} k(1-\gamma) Q_{i},
$$

we infer from (3.10) that

$$
\begin{gathered}
\gamma\left(\sum_{t \in N_{1}, t \neq i}\left|a_{i t}\right|+\omega_{t} \sum_{t \in N_{2}, t \neq i}\left|a_{i t}\right|+k \mu_{t} \sum_{t \in N_{3}, t \neq i}\left|a_{i t}\right|\right)+k\left(S_{j}-\mu_{j} \gamma \sum_{t \in N_{3}, t \neq i}\left|a_{j t}\right|\right)-\gamma\left(\sum_{t \in N_{1}, t \neq i}\left|a_{i t}\right|+\omega_{t} \sum_{t \in N_{2}, t \neq i}\left|a_{i t}\right|\right) \\
=\gamma\left(\sum_{t \in N_{1}, t \neq i}\left|a_{i t}\right|+\omega_{t} \sum_{t \in N_{2}, t \neq i}\left|a_{i t}\right|\right)+\gamma k \mu_{t} \sum_{t \in N_{3}, t \neq i}\left|a_{i t}\right|+k S_{j}-k \mu_{j} \gamma \sum_{t \in N_{3}, t \neq i}\left|a_{j t}\right|-\gamma\left(\sum_{t \in N_{1}, t \neq i}\left|a_{i t}\right|+\omega_{t} \sum_{t \in N_{2}, t \neq i}\left|a_{i t}\right|\right) \\
=k S_{j} \geq \gamma R_{i}(B)+(1-\gamma) Q_{i}(B) .
\end{gathered}
$$

Thus, we arrive that,

$$
\left|b_{i i}\right|=k \mu_{i}\left|a_{i i}\right|=k \frac{S_{i}}{\left|a_{i i}\right|}\left|a_{i i}\right|=k S_{i} \geq \gamma R_{i}(B)+(1-\gamma) Q_{i}(B) .
$$

Combined (i) - (iii) show that B is a strictly $\gamma$-diagonally dominant matrix, then B is a generalized strictly diagonally dominant matrix. By Lemma2.2, A is a generalized strictly diagonally dominant matrix, thus A is an H-matrix. This completes the proof.

Theorem 3.2 Let $A=\left(a_{i j}\right) \in \mathbb{C}^{n \times n}$, if $N_{1}, N_{2}, N_{3}$ satisfy $N=N_{1} \cup N_{2} \cup N_{3}$, then for $i \in N_{2}, j \in N_{3}$, there exists

$$
\begin{gathered}
\left(\omega_{i}\left|a_{i i}\right|-\left[\gamma\left(\sum_{t \in N_{1}, t \neq i}\left|a_{i t}\right|+r_{t} \sum_{t \in N_{2}, t \neq i}\left|a_{i t}\right|\right)+\omega_{i}(1-\gamma) Q_{i}\right]\right)\left(S_{j}-\left[\mu_{t} \gamma \sum_{t \in N_{3}, t \neq j}\left|a_{j t}\right|+\mu_{j}(1-\gamma) Q_{j}\right]\right) \\
>\left(\mu_{t} \gamma \sum_{t \in N_{3}, t \neq i}\left|a_{i t}\right|\right)\left(\gamma\left(\sum_{t \in N_{1}, t \neq j}\left|a_{j t}\right|+\omega_{t} \sum_{t \in N_{2}, t \neq j}\left|a_{j t}\right|\right)\right.
\end{gathered}
$$

and for $\forall i \in N(A)$, there exists a nonzero elements chain $a_{i i_{1}}, a_{i_{1} i_{2}}, \cdots, a_{i_{s} i_{m}}$, and $i \neq i_{1}, i_{1} \neq i_{2}, \cdots, i_{s} \neq i_{m}, i_{m} \in N(A) \neq \varnothing$, Thus $A \in G S D$, then $\mathrm{A}$ is a nonsingular H-matrix.

Proof. By the Theroem3.1, we also note that

$$
\begin{gathered}
d^{(1)}=\min \frac{\omega_{i}\left|a_{i i}\right|-\gamma \sum_{t \in N_{1}, t \neq i}\left|a_{i t}\right|-\omega_{t} \gamma \sum_{t \in N_{2}, t \neq i}\left|a_{i t}\right|-\omega_{i}(1-\gamma) Q_{i}}{\mu_{t} \gamma \sum_{t \in N_{3}, t \neq i}\left|a_{i t}\right|}, \\
d^{(2)}=\max \frac{\gamma\left(\sum_{t \in N_{1}, t \neq j}\left|a_{j t}\right|+\omega_{t} \sum_{t \in N_{2}, t \neq j}\left|a_{j t}\right|\right)}{S_{j}-\left[\mu_{t} \gamma \sum_{t \in N_{3}, t \neq j}\left|a_{j t}\right|+\mu_{j}(1-\gamma) Q_{j}\right]},
\end{gathered}
$$

we can get $d^{(1)}>d^{(2)}$, then we take any $\mathrm{k}$ and assume that $\min d^{(1)} \geq k \geq \max d^{(2)}$, we suppose

$$
\begin{gathered}
D=\operatorname{diag}\left\{d_{1}, d_{2}, \cdots, d_{n}\right\}, \\
d_{i}=\left\{\begin{array}{c}
1, i \in N_{1} \\
\omega_{i}, i \in N_{2} \\
k \mu_{i}, i \in N_{3}
\end{array}\right.
\end{gathered}
$$

We suppose

$$
B=A D=\left(b_{i j}\right),
$$

In a similar way to the proof of Theorem3.1, we know that matrix B is a $\gamma$-diagonally dominant matrix.

For $\mathrm{D}$ is a positive diagonally matrix, when A satisfies the condition of lemma2.3, further $\forall i \in N(A)$, there exists a nonzero elements chain $b_{i i_{1}}, b_{i_{1} i_{2}}, \cdots, b_{i_{s} i_{m}}$, and $i \neq i_{1}, i_{1} \neq i_{2}, \cdots, i_{s} \neq i_{m}, i_{m} \in N \backslash N(A) \neq \varnothing$.

By the lemma2.2, thus B is a generalized diagonally dominant matrix, then A is a nonsingular H-matrix. The proof is completed. 


\section{Example 1 Let}

$$
A=\left[\begin{array}{cccc}
10 & 4 & 2 & 1 \\
3 & 10 & 1 & 2 \\
4 & 5 & 9 & 4 \\
8 & 4 & 5 & 10.6
\end{array}\right], \gamma=\frac{1}{2}
$$

Take

$$
N_{1}=\varnothing, N_{2}=\{1,3,4\}, N_{3}=\{2\},
$$

Then

$$
S_{1}=11, S_{2}=9.5, S_{3}=10.5, S_{4}=12 .
$$

According to Theorem3.1, we obtain

$$
\begin{aligned}
& \left(\omega_{1}\left|a_{11}\right|-\left[\frac{1}{2}\left(\omega_{3}\left|a_{13}\right|+\omega_{4}\left|a_{14}\right|\right)+\frac{1}{2} \omega_{1} Q_{1}\right]\right)\left(S_{2}-\frac{1}{2} \mu_{2} Q_{2}\right)=1.2202 \\
& >\left(\frac{1}{2} \mu_{2}\left|a_{12}\right|\right)\left(\frac{1}{2}\left(\omega_{1}\left|a_{21}\right|+\omega_{3}\left|a_{23}\right|+\omega_{4}\left|a_{24}\right|\right)\right)=0.6165, \\
& \left(\omega_{3}\left|a_{33}\right|-\left[\frac{1}{2}\left(\omega_{1}\left|a_{31}\right|+\omega_{4}\left|a_{34}\right|\right)+\frac{1}{2} \omega_{3} Q_{3}\right]\right)\left(S_{2}-\frac{1}{2} \mu_{2} Q_{2}\right)=1.945 \\
& >\left(\frac{1}{2} \mu_{2}\left|a_{32}\right|\right)\left(\frac{1}{2}\left(\omega_{1}\left|a_{21}\right|+\omega_{3}\left|a_{23}\right|+\omega_{4}\left|a_{24}\right|\right)\right)=0.7706, \\
& \left(\omega_{4}\left|a_{44}\right|-\left[\frac{1}{2}\left(\omega_{1}\left|a_{41}\right|+\omega_{3}\left|a_{43}\right|\right)+\frac{1}{2} \omega_{4} Q_{4}\right]\right)\left(S_{2}-\frac{1}{2} \mu_{2} Q_{2}\right)=1.0365 \\
& >\left(\frac{1}{2} \mu_{2}\left|a_{42}\right|\right)\left(\frac{1}{2}\left(\omega_{1}\left|a_{21}\right|+\omega_{3}\left|a_{23}\right|+\omega_{4}\left|a_{24}\right|\right)\right)=0.6165 .
\end{aligned}
$$

Thus matrix A satisfies the conditions of the Theorem3.1, then By Theorem3.1, matrix A is an H-matrix. According to the method of comparing matrix element in reference[2], we have

$$
\begin{aligned}
& \left|a_{33}\right|=9 \\
& <\frac{\Lambda_{3}(A)}{\Lambda_{3}(A)-\left|a_{33}\right|}\left[\frac{\Lambda_{1}(A)-\left|a_{11}\right|}{\Lambda_{1}(A)}\left|a_{31}\right|+\frac{\Lambda_{4}(A)-\left|a_{44}\right|}{\Lambda_{4}(A)}\left|a_{34}\right|+\frac{\Lambda_{2}(A)-\left|a_{22}\right|}{\Lambda_{2}(A)}\left|a_{32}\right|\right] \\
& =9.0727 .
\end{aligned}
$$

In reference[2], we can not obtain the conclusion that matrix A is an H-matrix. This shows that the method given in this paper is more practical in judging $\mathrm{H}$-matrix.

\section{Conclusion}

In this paper, two new helpful definitions of strong and weak diagonal dominance degree and the sum of non-principal diagonal element for the matrix are given. Further, we present a new method of judging H-matrix. For making up for the deficiency of the existing literatures such as reference[2], as a contrast, our method can be used to judge and widen the range of conditions for judging $\mathrm{H}$-matrix. Moreover, numerical example shows that the criterion is practical and feasible.

\section{Acknowledgements}

This research is supported by the Hujiang Foundation of China (B14005).

\section{References}

Cvetkovic, L., \& Kostic, V. (2005). New criteria for identifying H-matrices. Journal of Computational and Applied Mathematics, 180(2), 265-278. https://doi.org/10.1016/j.cam.2004.10.017

Gan, T. B., \& Huang, T. Z. (2004). Practical sufficient conditions for nonsingular H-matrices. Mathematica Numerica Sinica, 26(1), 109-116. https://dx.doi.org/10.2116/analsci.20.717

Gao, H. S., \& Han, G. C. (2017). New Criteria for Nonsingular H-Matrices and Its Applications. 2017 4th International Conference on Information Science and Control Engineering (ICISCE). IEEE Computer Society. https://doi.org/10.1016/0024-3795(95)00251-0

Gao, Y. M., \& Wang, X. H. (1996). Criteria for generalized diagonally dominant matrices and M-matrices. Journal Of Mathematical Research and Exposition, 248(4), 339-353. https://doi.org/10.1016/0024-3795(95)00251-0 
Huang, T. Z., Leng, J. S., Wachspress, E. L., \& Tang, Y. Y. (2004). Characterization of H-matrices. Computers and Mathematics with Applications, 48(10-11), 1587-1601. https://doi.org/10.1016/j.camwa.2004.04.034

Jeribi, A., Krichen, B., \& Zitouni, A. (2019). Spectral properties for $\gamma$-diagonally dominant operator matrices using demicompactness classes and applications. Revista de la Real Academia de Ciencias Exactas, Fisicas y Naturales. Serie A. Matematicas. https://doi.org/10.1007/s13398-019-00629-0

Li, G. Q., Liu, J. Z., \& Zhang, J. (2017). The disc theorem for the schur complement of two class submatrices with $\gamma$-diagonally dominant properties. Numerical Mathematics: Theory, Methods and Applications, 10(1), 84-97. https://doi.org/10.4208/nmtma.2017.y14034

Li, M., \& Sun, Y. X. (2009). Practical criteria for H-matrices. Applied Mathematics and Computation, 211(2), 427-433. https://doi.org/10.4208/nmtma.2017.y14034

Liu, J. Z., \& Huang, Y. Q. (2004). Some properties on schur complements of H-matrices and diagonally dominant matrices. Linear Algebra and its Applications, 389(none), 365-380. https://doi.org/10.1016/j.laa.2004.04.012

Liu, J. Z., \& He, A. Q. (2007). An interleaved iterative criterion for H-matrices. Applied Mathematics and Computation, 186(1), 727-734. https://dx.doi.org/10.1016/j.amc.2006.08.031

Liu, J. Z., \& Huang, Z. J. (2010). The schur complements of $\gamma$-diagonally and product $\gamma$-diagonally dominant matrix and their disc separation. Linear Algebra and its Applications, 432(4), 1090-1104. https://dx.doi.org/10.1016/j.laa.2009.10.021

Liu, J. Z., Zhang, J., Zhou, L. X., \& Tu, G. (2018). The Nekrasov diagonally dominant degree on the Schur complement of Nekrasov matrices and its applications. Applied Mathematics and Computation, 320, 251-263. https://dx.doi.org/10.1016/j.amc.2017.09.032

Ostrowski, A. M. (1937). Uber die determinant mit uberweigenber hauptdiagonale. Comment Math, 10, 69-96.

Varga, R. S. (1976). On recurring theorems on diagonal dominance. Linear Algebra and its Applications, 13(1-2), 1-9. https://dx.doi.org/10.1016/0024-3795(76)90037-9

Xie, Q. M., He, A. Q., \& Liu, J. Z. (2007). On the iterative method for H-matrices. Applied Mathematics and Computation, 189(1), 41-48. https://dx.doi.org/10.1016/j.amc.2006.11.133

Zhu, H. T. (1995). A note on generalized diagonally dominant matrices. Linear Algebra and its Applications, 225(225), 237-242. https://dx.doi.org/10.1016/0024-3795(93)00368-A

\section{Copyrights}

Copyright for this article is retained by the author(s), with first publication rights granted to the journal.

This is an open-access article distributed under the terms and conditions of the Creative Commons Attribution license (http://creativecommons.org/licenses/by/4.0/). 